\title{
Electrical Imaging of the Changes in Hyporheic Exchange from Channel-Spanning Logjams
}

By Megan Doughty ${ }^{1}$, Ethan Ader ${ }^{2}$, Jackie Randell ${ }^{1}$, Ellen Wohl ${ }^{2}$, and Kamini Singha ${ }^{1}$ 


\section{Background}

$\square$ Human impacts alter natural processes and features of streams

Timber harvesting

- Stream engineering

- Beaver removal

- Urbanization

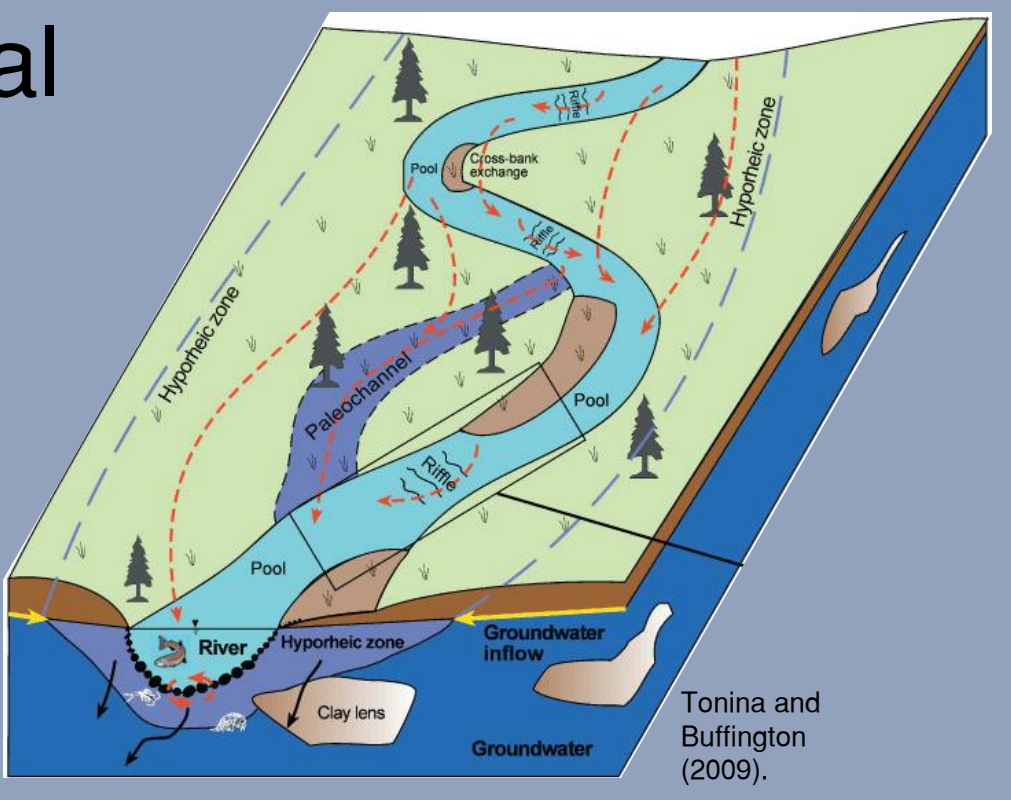

$\square$ Natural heterogeneity is important for sediment transportation and healthy ecosystems 


\section{Logjams}

$\square$ Logjams increase hydraulic resistance and create pressure gradients driving hyporheic exchange flow

- Transfers dissolved oxygen, solutes, and nutrients

- Regulates temperature fluctuations

$\square$ Creates diverse habitats

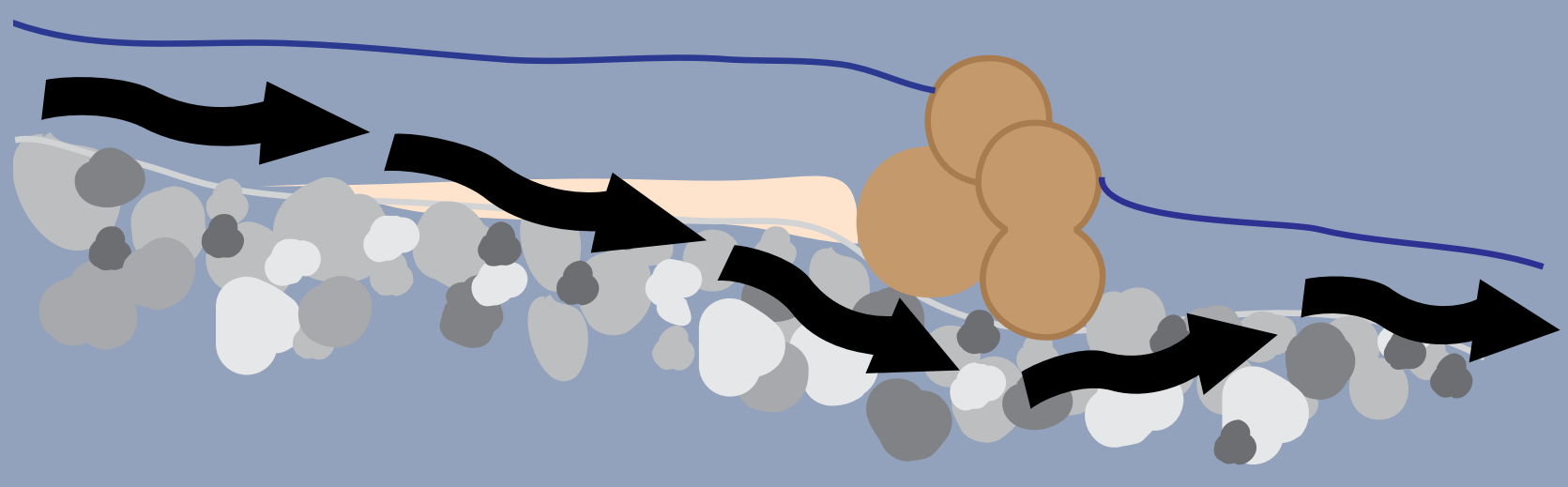




\section{Field Site: Little Beaver Creek}

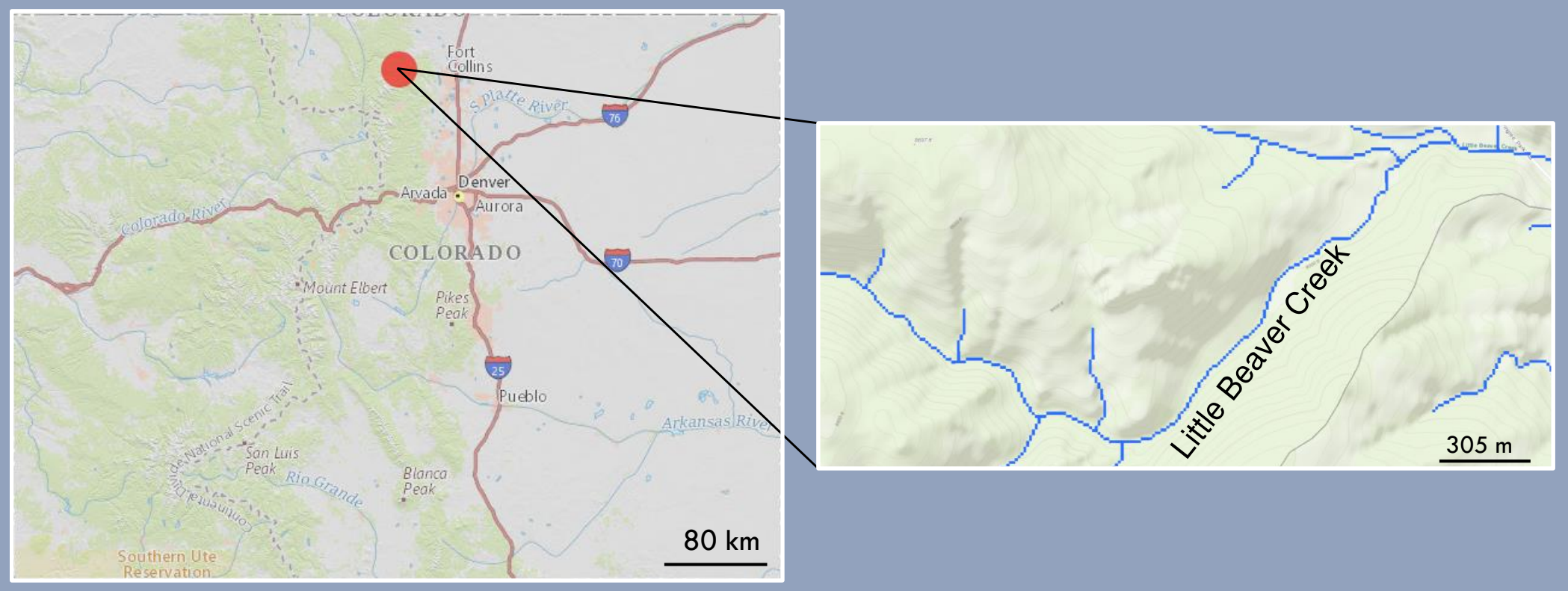

$\square \quad$ Drainage Area: 40 km²

Third-order tributary to the South Fork of the Cache la Poudre River

Montane Forest (1,840-2,740 m elevation)

Snowmelt Dominated

Abundant logjams and minimum human alteration 


\section{Logjam Site}

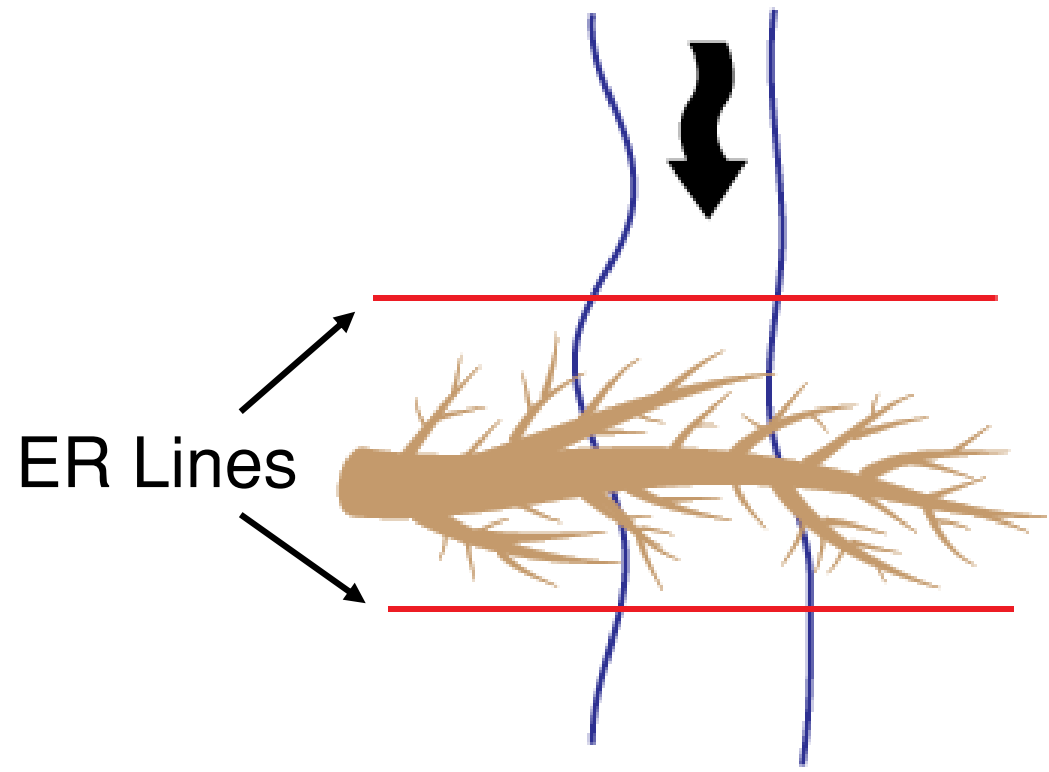

Map View
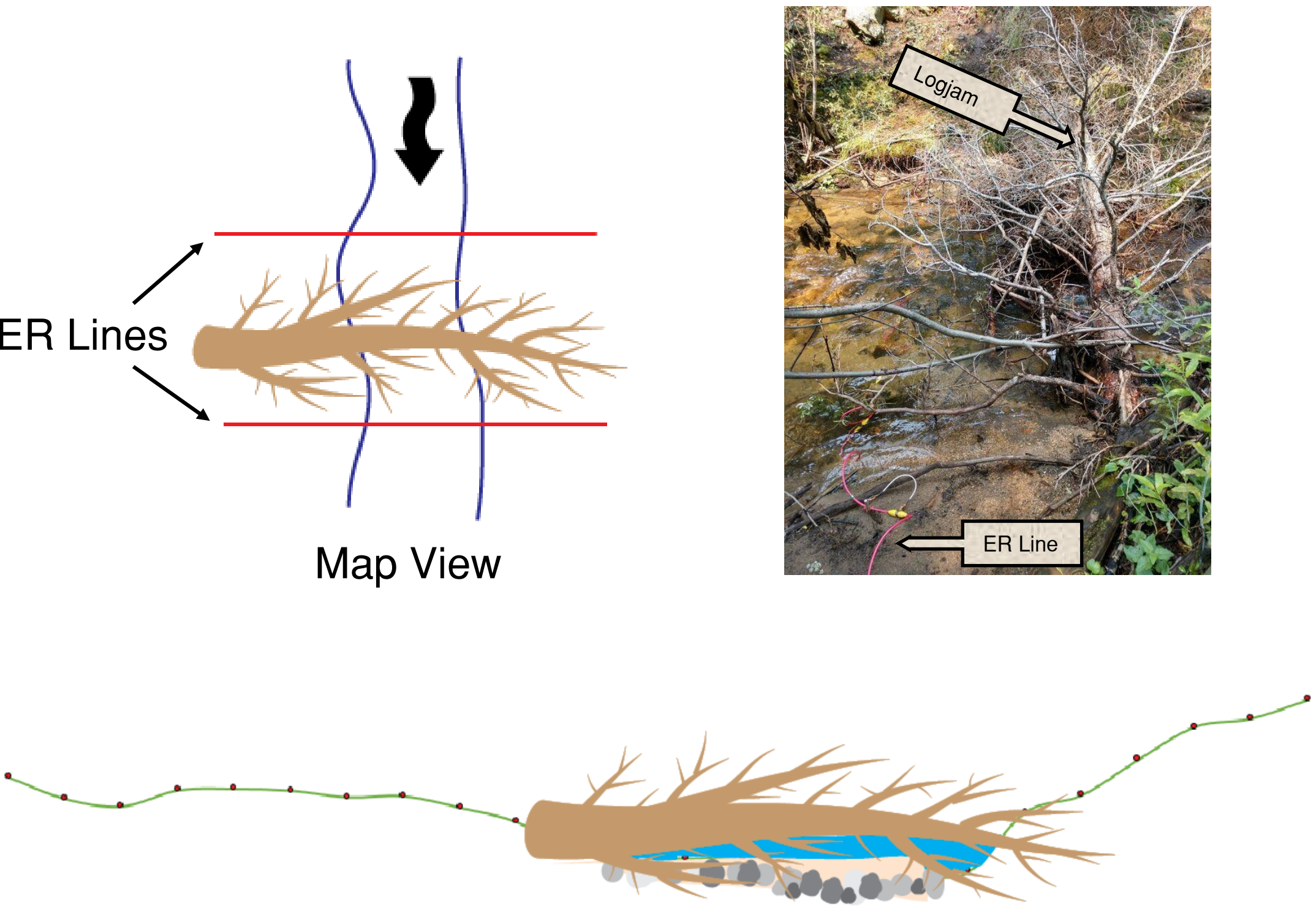

Cross-Section 


\section{Control Site}
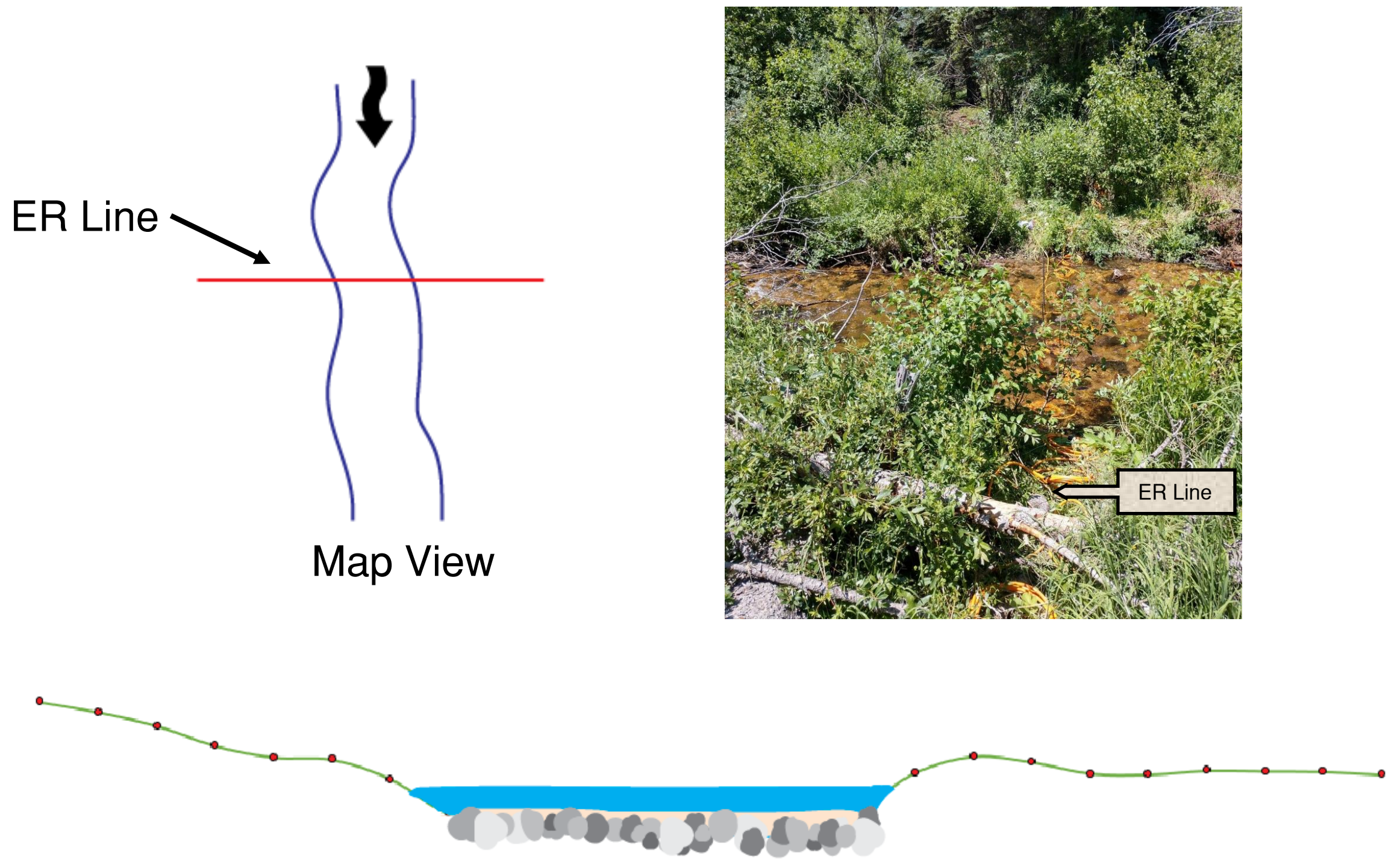

\section{Map View}

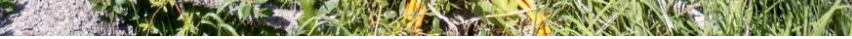

Cross-Section 


\section{Field Methods}

$\square$ 4-hour injection of a salt tracer

$\square \mathrm{NaCl}$ is highly conductive

$\square \quad$ In-stream and ER monitoring for 24 hours

24 electrodes with 1 to 0.5 meter spacing

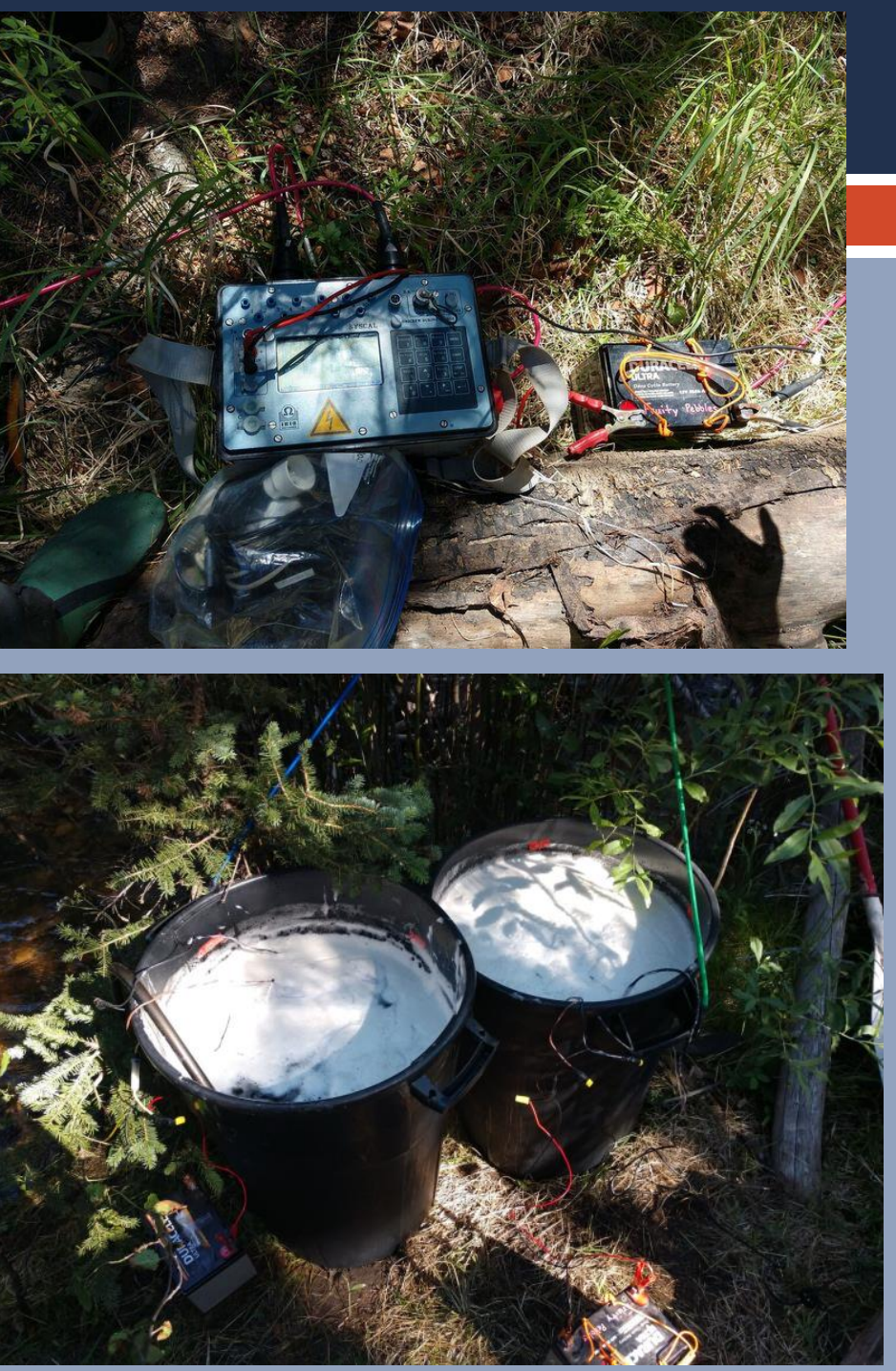




\section{Field Methods: Electrical Resistivity}

$\square \quad$ ER passes low-frequency alternating current along the lines

$\square \quad$ Typical solute monitoring methods (i.e. wells and in-stream) are insufficient to capture hyporheic exchange

$\square$ ER provides spatial and temporal data
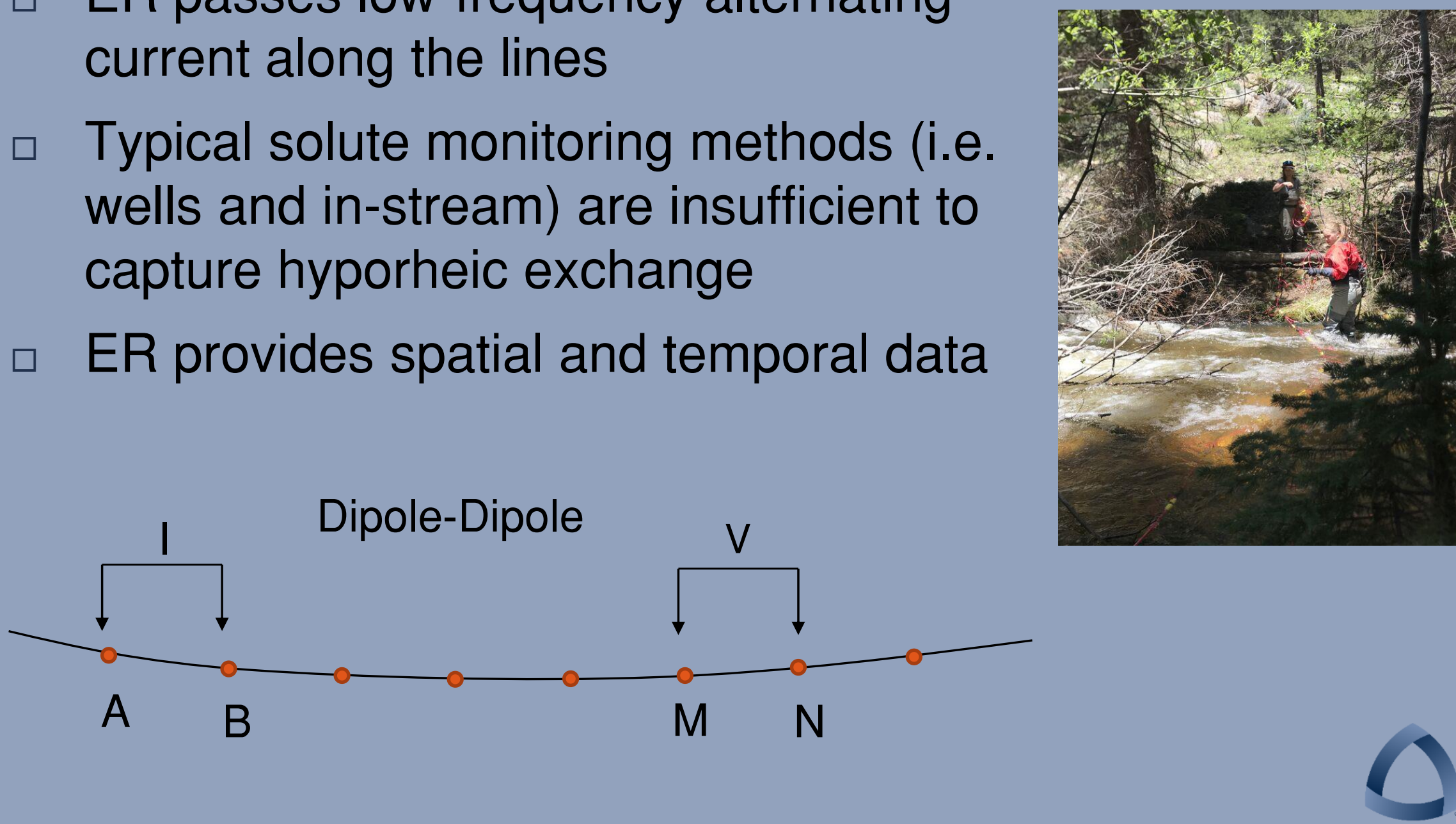
Calculated Discharge (cms) Based on Rating Curve

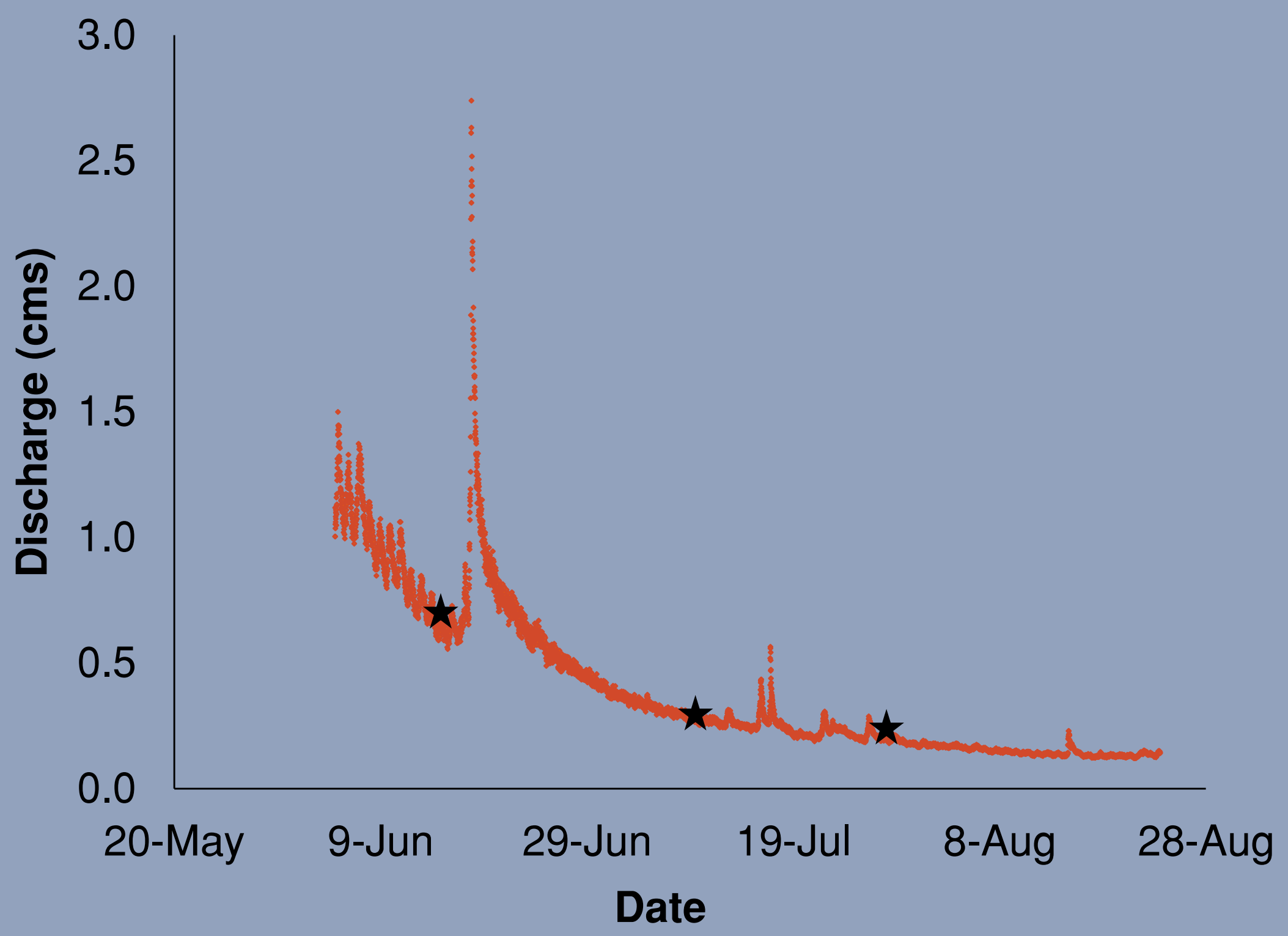




\section{Background Resistivity}
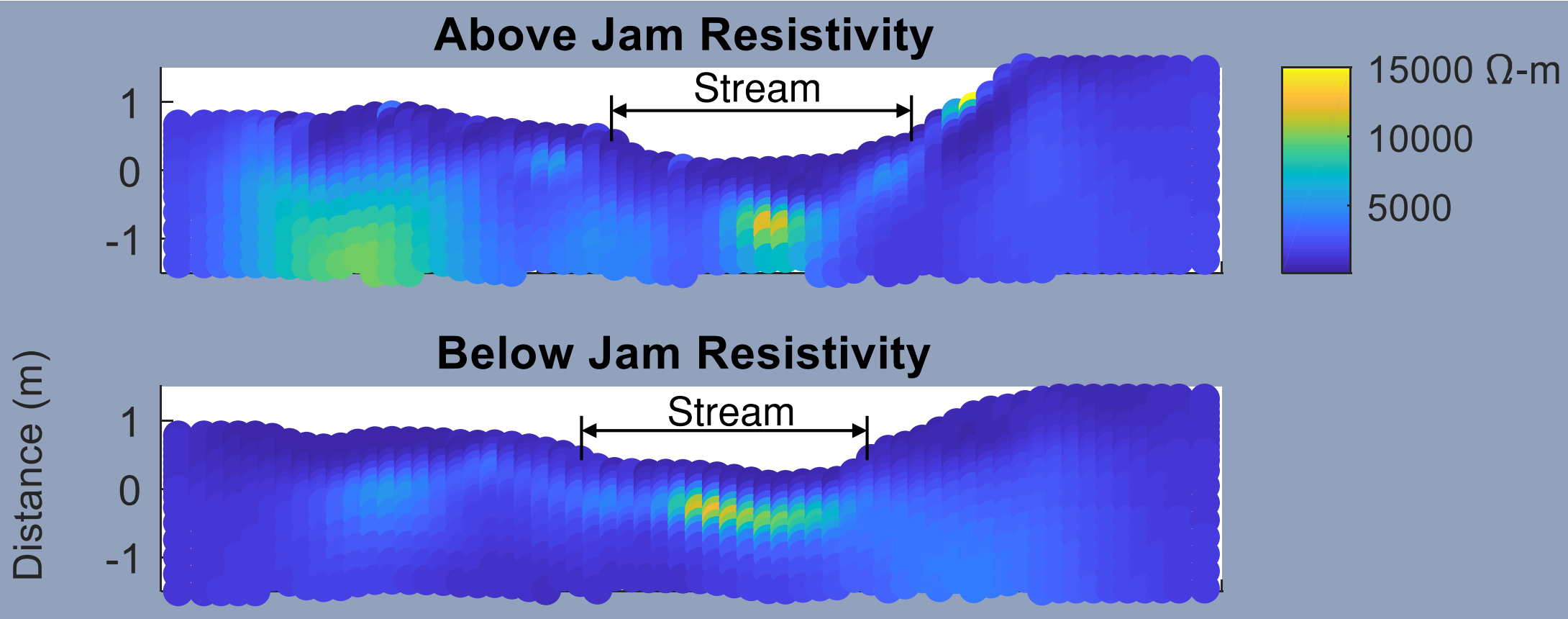

Control Resistivity

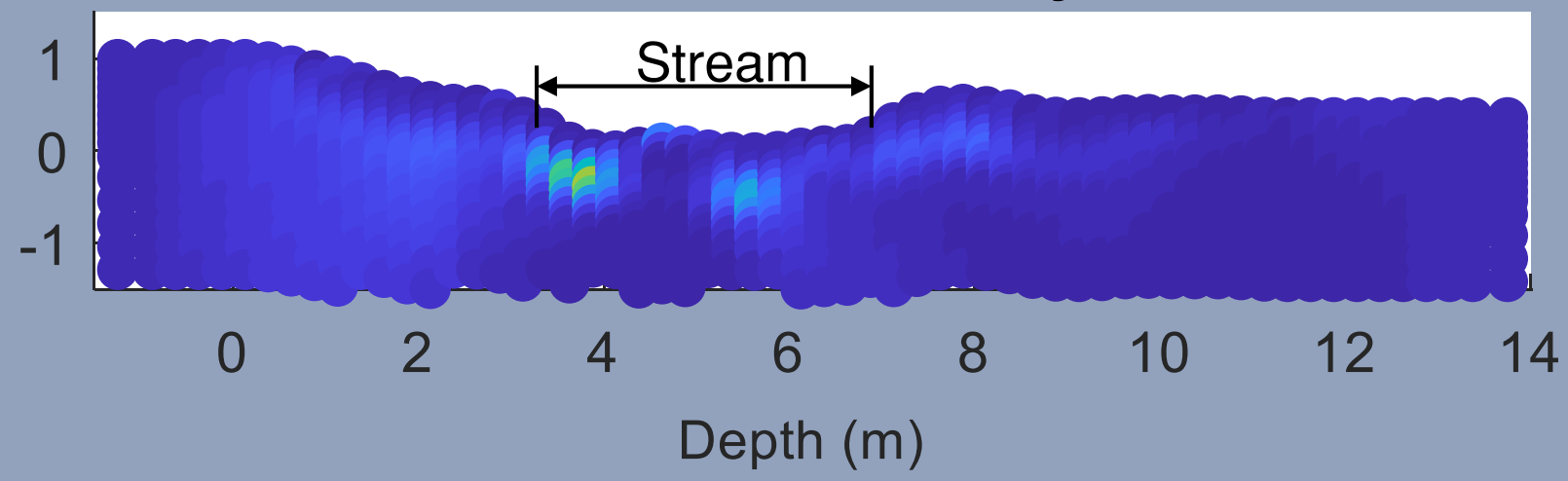

Background Fluid Resistivity : $416 \Omega-\mathrm{m}$ (EC: 24 uS/cm) 


\section{Resolution Matrix}

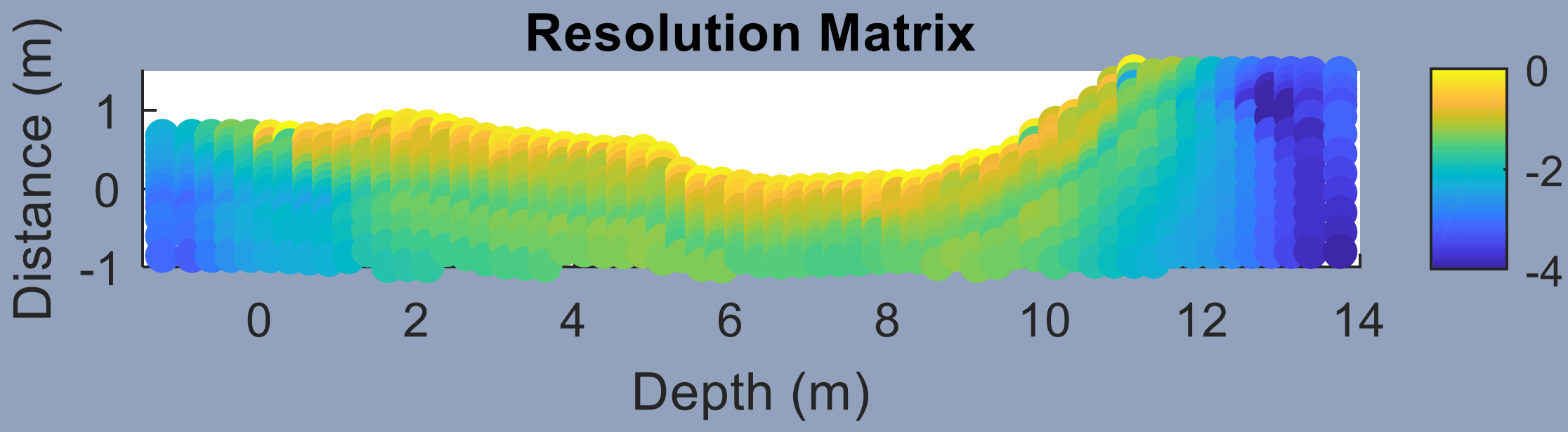

Log10(diagonal of resolution matrix)

$\square$ Pixels equal to $\log (1)=0$ have perfect resolution

$\square \quad$ Pixels less than 1 (negative in log space) have less than perfect resolution 


\section{Logjam Site: Changes in ER}

Above Jam During Tracer Injection

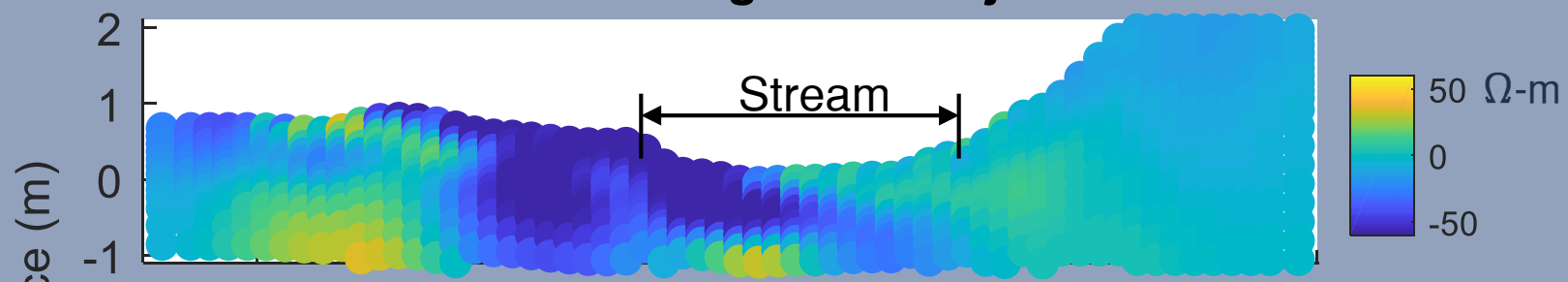

Above Jam After Tracer Injection

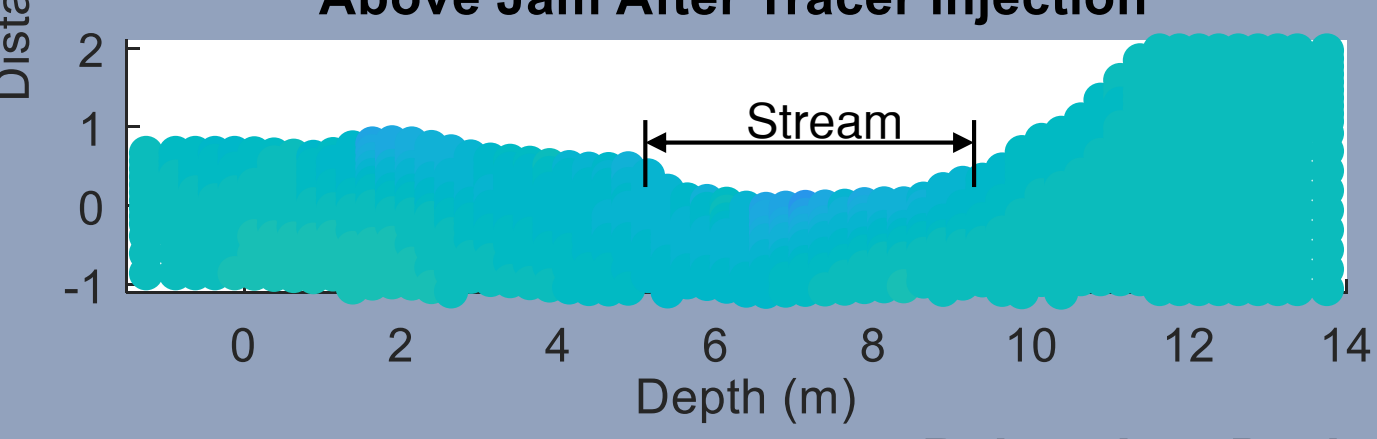

Below Jam During Tracer Injection
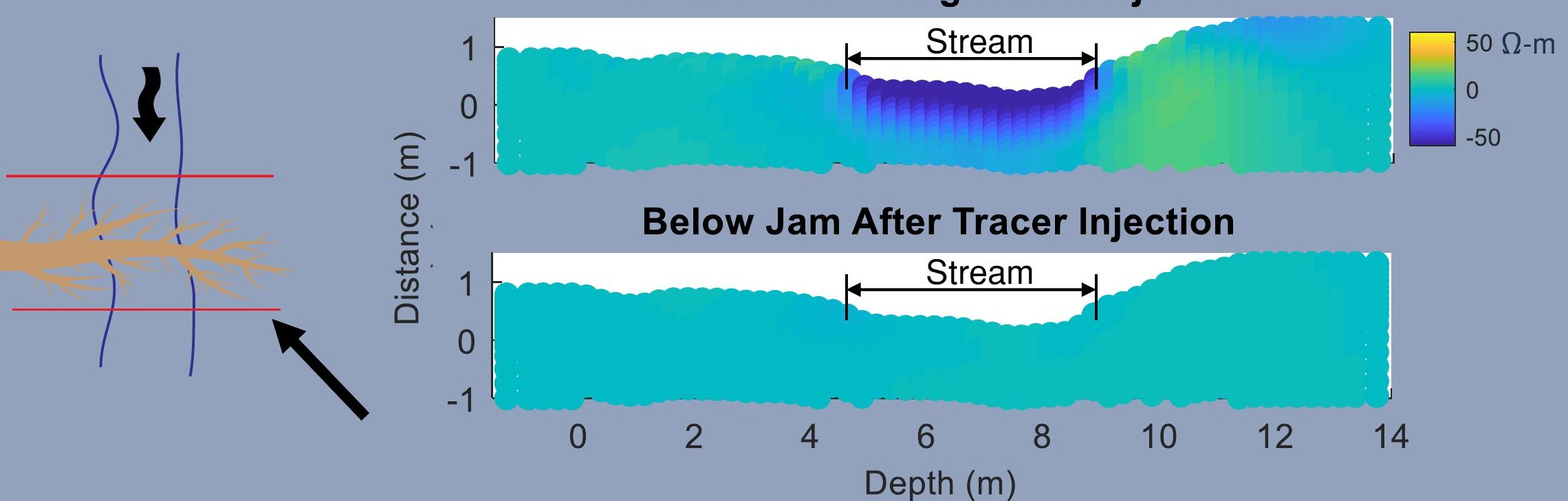


\section{Control Site: Changes in ER}

Control During Tracer Injection

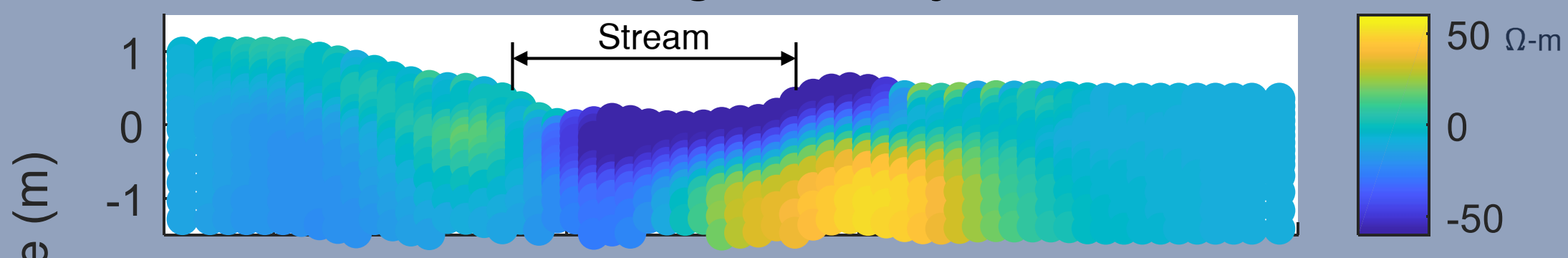

Control During Tracer Injection

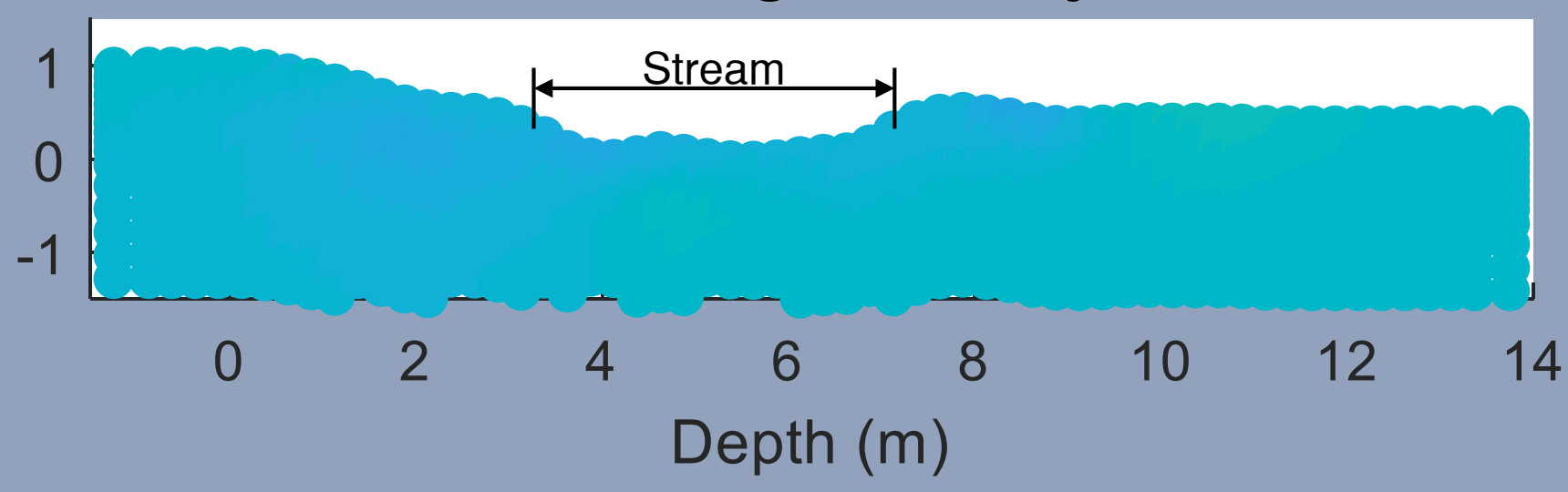




\section{Changes in ER Above Logjam Over Time}

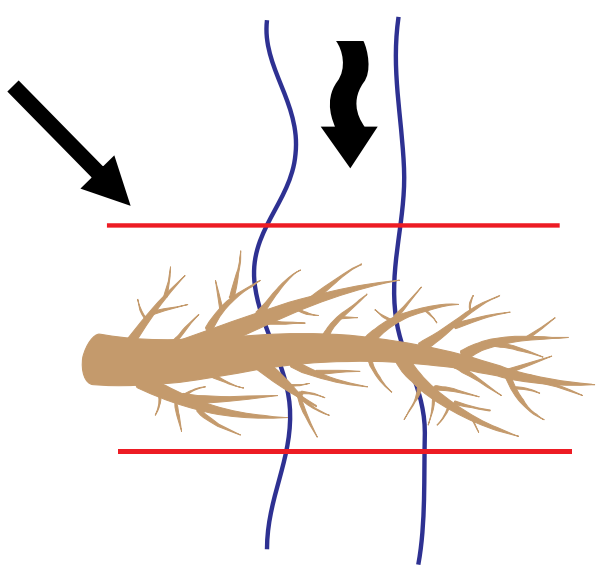

Above Jam Tracer Test 6/13: timestep 12

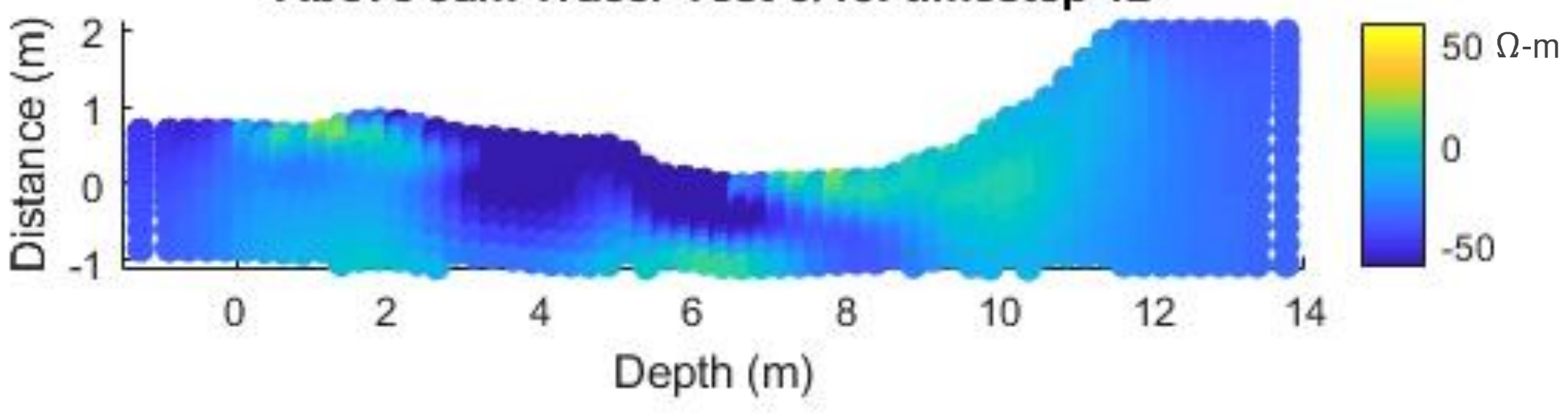




\section{Tracer Test 6/13-14}

\section{Fluid vs Bulk Electrical Conductivity}

Control 6/13-14

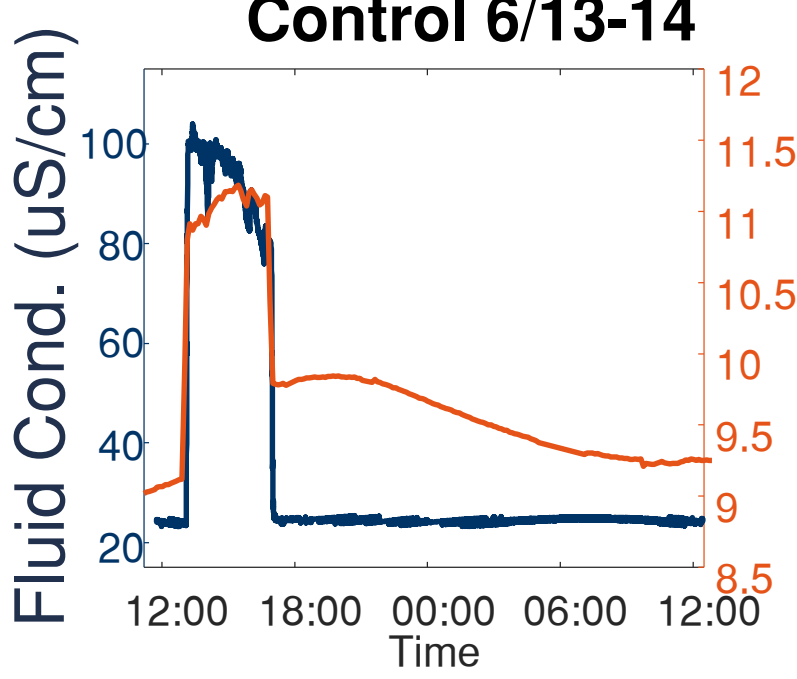

Above Jam 6/13-14

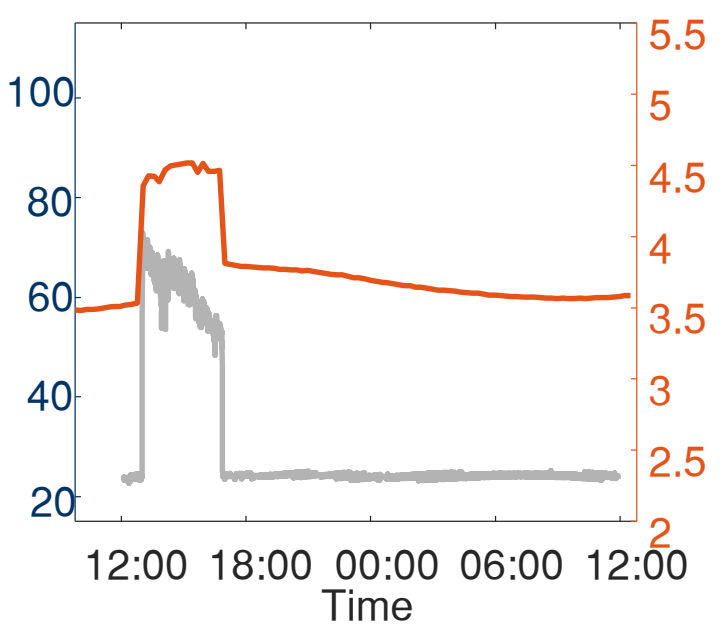

Below Jam 6/13-14

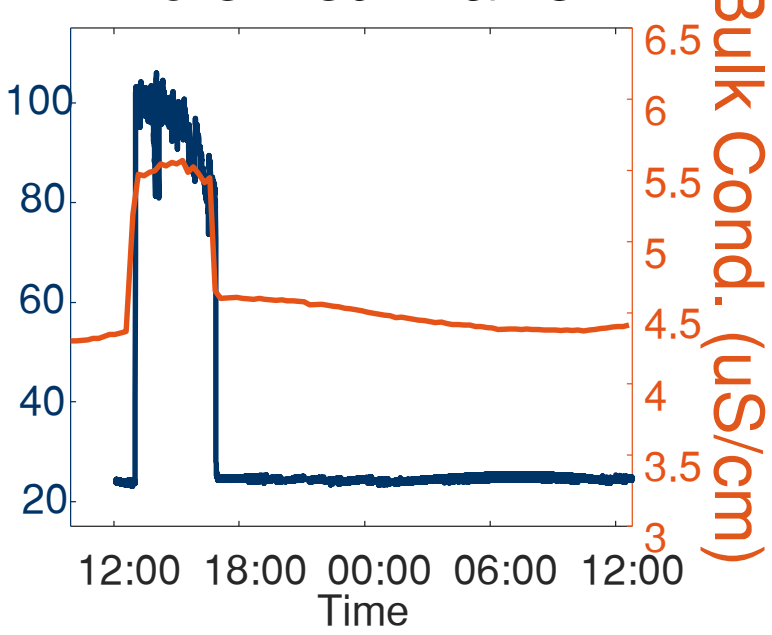

\begin{tabular}{|c|c|c|c|c|c|c|}
\hline Tracer 6/13-14 & \multicolumn{2}{|c|}{ Control } & \multicolumn{2}{c|}{ Above Jam } & \multicolumn{2}{c|}{ Below Jam } \\
\hline Mean Arrival Time $(\mathrm{hr})$ & 7 & 2 & 6 & 3 & 6 & 2 \\
\hline Variance $\left(\mathrm{hr}^{2}\right)$ & 36 & 5 & 36 & 15 & 34.0 & 10 \\
\hline Skewness $\left(\mathrm{hr}^{3}\right)$ & 220 & 57 & 254 & 188 & 271 & $\mathbf{1 3 5}$ \\
\hline
\end{tabular}




\section{Flow Comparisons}

\begin{tabular}{|l|l|l|}
\hline Tracer 6/13-14 & Bulk & Fluid \\
\hline Mean Arrival Time (hr) & 6 & 2 \\
\hline Variance $\left(\mathrm{hr}^{2}\right)$ & 34 & 10 \\
\hline Skewness $\left(\mathrm{hr}^{3}\right)$ & $\mathbf{2 7 1}$ & $\mathbf{1 3 5}$ \\
\hline
\end{tabular}

Discharge: $0.86 \mathrm{cms}$

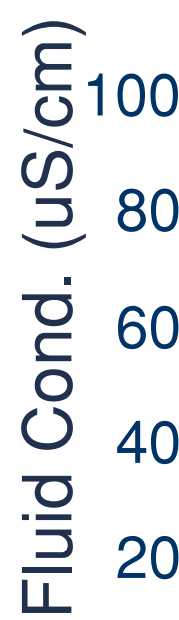

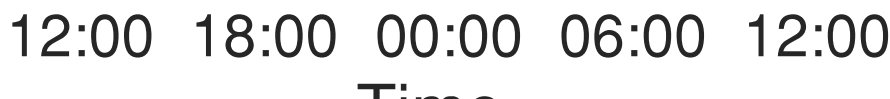
Time
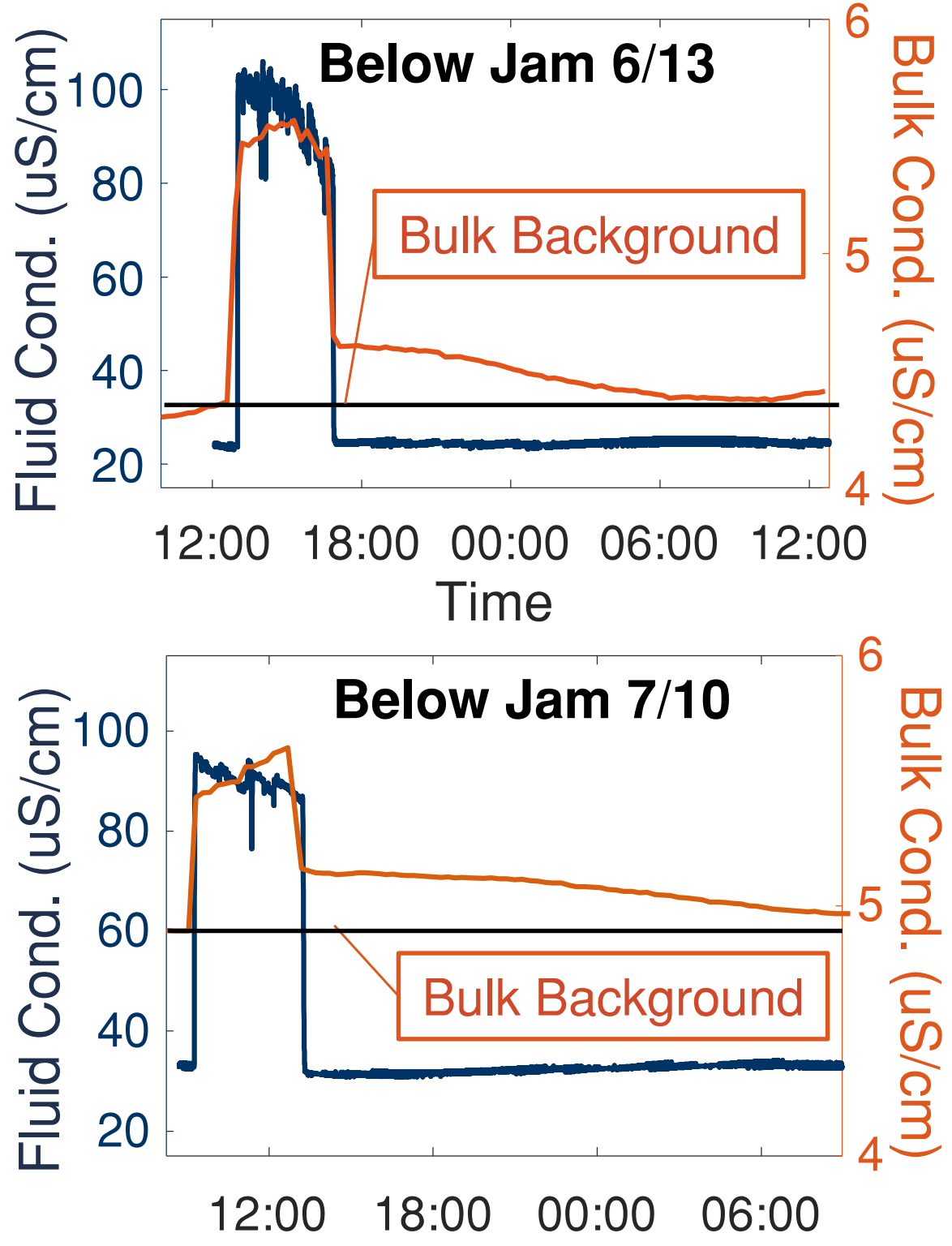

Time

\begin{tabular}{|l|l|l|}
\hline Tracer $7 / 10-11$ & Bulk & Fluid \\
\hline Mean Arrival Time $(\mathrm{hr})$ & 7 & 2 \\
\hline Variance $\left(\mathrm{hr}^{2}\right)$ & 40 & 9 \\
\hline Skewness $\left(\mathrm{hr}^{3}\right)$ & 193 & $\mathbf{1 3 5}$ \\
\hline
\end{tabular}

Discharge: $0.17 \mathrm{cms}$ 


\section{Conclusions}

\section{Applications}

$\square \quad$ ER improves measurements of the hyporheic zone

$\square$ Logjams increase hyporheic exchange flow

$\square$ Higher discharge rates increase hyporheic exchange flow $\square$ Characterize human impacts on stream functions

$\square \quad$ Improve water quality

- Advance stream restoration and conservation efforts 


\section{Acknowledgements}

$\square \quad$ NSF (award no. 1819134)

$\square \quad$ Colorado School of Mines and Colorado State University

- All of my wonderful help in the field:

- Jackie Randell, Teodora Mitroi, James Proctor, Ethan Ader, Emily Iskin, Ellen Wohl, Kamini Singha, Audrey Sawyer, Kenneth Swift Bird, Amelia Nelson, Rachel Corrigan, Mihri Genc, and more!
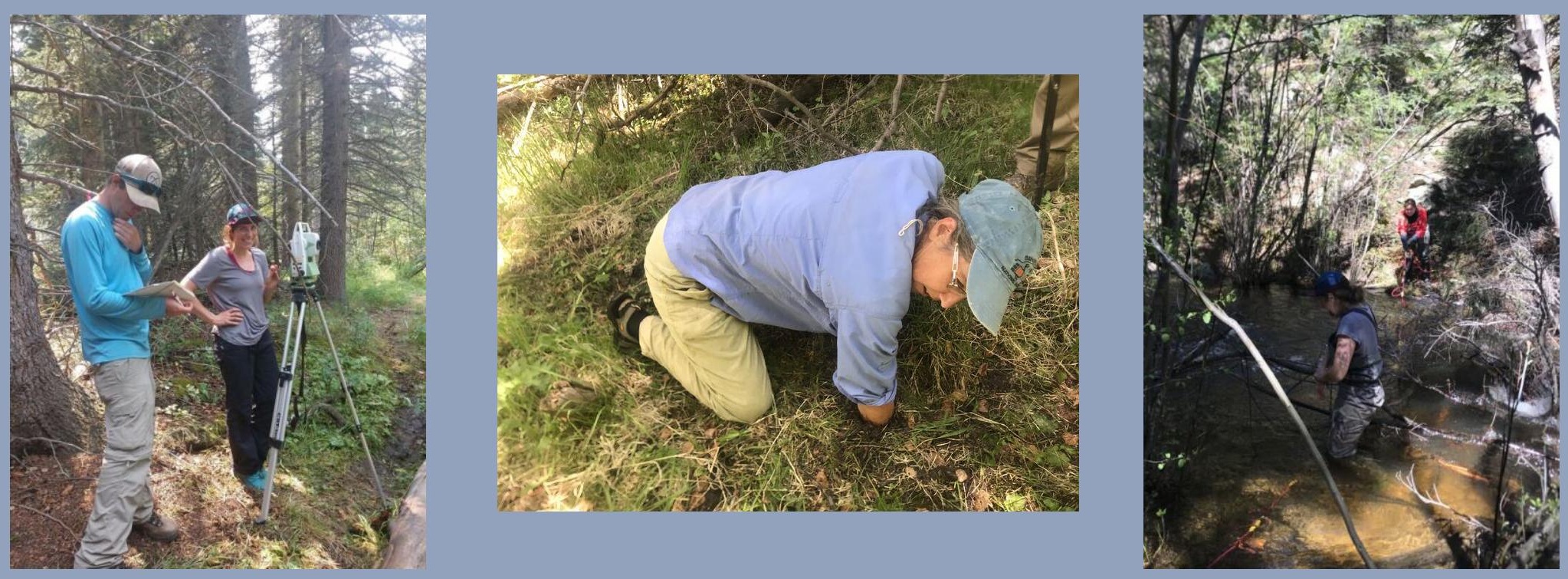


\section{Questions?}
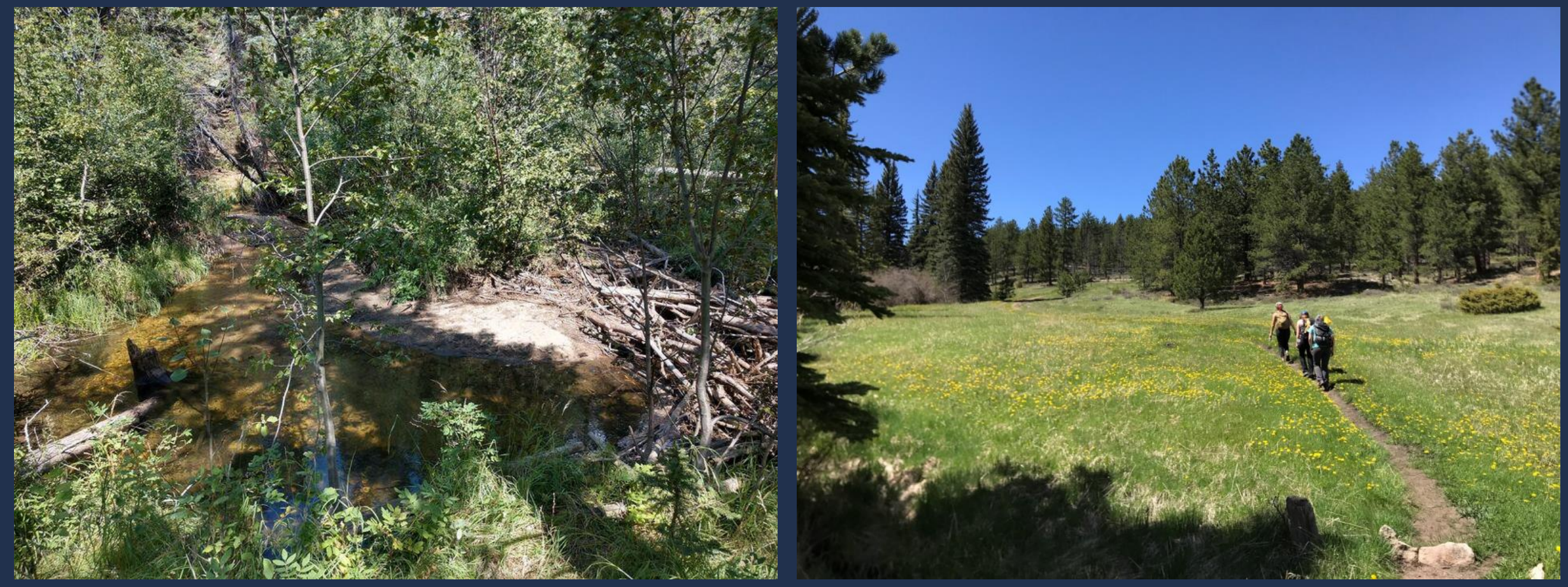